\title{
Assessment of Left Ventricular Outflow in Hypertrophic Cardiomyopathy Using Anyplane and Paraplane Analysis of Three-Dimensional Echocardiography
}

\author{
Alessandro Salustri, MD, PhD, Marcel J.M. Kofflard, MD, Jos R.T.C Roelandt, MD, PhD, \\ Youssef Nosir, MD, Giuseppe Trocino, MD, David Keane, MB, MRCPI, Wim B. Vletter, BSC, \\ and Folkert J. Ten Cate MD, PhD
}

This study analyzes the alterations in size and geometry of the left ventricular (LV) outflow tract that occur in hypertrophic cardiomyopathy $(\mathrm{HC})$ using transthoracic 3dimensional echocardiography. Transthoracic 3-dimensional echocardiography was performed in 17 patients with HC (4 after myectomy) and in 10 normal subjects. Images were acquired with the rotational approach, with electrocardiographic and respiratory gating. From the 3-dimensional datasets, short-axis parallel slicing of the LV outflow tract at a $1 \mathrm{~mm}$ distance was performed at the onset of systole. For each slice, cross-sectional area and maximal and minimal diameter were calculated. Reconstruction of the LV oufflow tract could be displayed in 3 dimensions in all patients, allowing orientation and clear definition of the irregular geometry. In patients with HC, the minimal LV oufflow tract crosssectional area was smaller than in normal subjects (2.3

$\rightarrow$ ost ostmortem studies and intraoperative findings indicate that 2-dimensional echocardiography may fail to give the full picture of the left ventricular (LV) outflow tract in patients with hypertrophic cardiomyopathy (HC). ${ }^{1-6}$ Three-dimensional echocardiography is a new imaging modality which provides unique information on the spatial geometry of a given structure. ${ }^{7}$ Based on our experience in an unselected population, ${ }^{8,9}$ we believe that the alterations in size and geometry of the LV outflow tract in patients with $\mathrm{HC}$ could be more accurately analyzed with precordial 3-dimensional echocardiography. With these concepts in mind, we designed this study to gain further insight into the complex geometry of the LV outflow tract in patients with HC. With this aim, analysis of the LV outflow tract was performed using 3-dimensional datasets obtained after acquisition of precordial echocardiographic images.

\footnotetext{
From the Department of Cardiology, Thoraxcenter, University Hospita Rotterdam-Dijkzigt and Erasmus University, Rotterdam, The Netherlands. Dr. Nosir is supported by the Nuffic, The Netherlands, and the Department of Cardiology, Al-Azhar University Hospital, Cairo, Egypt. Dr Keane is the recipient of a travel grant from Peel Medical Research Trust, London, United Kingdom. Manuscript received November 20, 1995; revised manuscript received and accepted February 20,1996

Address for correspondence: Folkert Ten Cate, MD, Thoraxcenter, Ba 350, Dr. Molewaterplein, 40, 3015 GD Rotterdam, The Netherlands.
}

$\left.\pm 1.0 \mathrm{vs} 5.0 \pm 0.9 \mathrm{~cm}^{2}, p<0.0001\right)$. The ratio between maximal and minimal cross-sectional areas was higher in patients with $\mathrm{HC}$ than in normal subjects $(2.6 \pm 0.9$ vs $1.4 \pm 0.2, p<0.0001$ ). The ratio between maximal and minimal diameter of the smallest cross section of the LV oufflow tract was also significantly higher in patients with $\mathrm{HC}$ than in normal subjects ( $1.6 \pm 0.3$ vs $1.2 \pm 0.1$, $\mathrm{p}<0.001)$; a value of 1.36 separated normal subjects from HC patients without previous myectomy. In conclusion, precordial 3-dimensional echocardiography allows detailed qualitative and quantitative information on the LV outflow tract. Patients with HC are characterized by a highly eccentric and asymmetric shape of the IV oufflow tract, and by a smaller minimal cross-sectional area than that seen in normal subjects.

(Am J Cordiol 1996;78:462-468)

\section{METHODS}

Study patients: We prospectively selected 17 patients ( 13 men and 4 women; mean \pm SD age $39 \pm$ 15 years, range 19 to 65 ) with $\mathrm{HC}$ referred to the outpatient clinic of our institution for routine transthoracic echocardiographic follow-up. High-quality images were a prerequisite for inclusion in this study. The diagnosis of $\mathrm{HC}$ was based on M-mode and 2dimensional echocardiographic demonstration of a nondilated hypertrophic left ventricle in the absence of other cardiac or systemic disease that could produce LV hypertrophy. ${ }^{10}$ According to a previously established classification, ${ }^{11}$ the patterns of distribution of LV hypertrophy were: type I, 1 patient; type II, 3 patients; type III, 12 patients; and type IV, 1 patient. Systolic anterior motion of the mitral valve was present in 12 patients and its severity was evaluated semiquantitatively from $0=$ absence, to $3+=$ contact with the interventricular septum during systole. $^{5}$ At the time of the echocardiographic study, a pressure difference was calculated from Doppler LV outflow tract velocity recordings, and obstruction (gradient $>30 \mathrm{~mm} \mathrm{Hg}$ under basal conditions) was detected in 4 patients. A septal myectomy had been performed in 4 patients. Ten asymptomatic subjects without evidence of LV hypertrophy were also studied for comparison. These controls were aged 20 to 49 years (mean $28 \pm 8$ ) and 8 were men.

Examination procedure: Two-dimensional echocardiographic studies were performed with a commer- 
FIGURE 1. Analysis of left ventricular (LV) outflow tract in a patient with hypertrophic cardiomyopathy. From the 3-dimensional datuset, parallel slicing of the LV outflow tract perpendicular to the long axis (paraplane echocordiography) is performed at $1 \mathrm{~mm}$ in tervals, at the onset of ventricular systole. In the loft ponel, 3 representative cut-planes are indicated. The corresponding cross-sectional 2-dimensional images with the manually traced endocardial contours are shown in the middle panels. The final wire frame mode display represented in the right panel can be rotated on the screen along the 3 main axes for versatile qualitative 3-dimensional evaluation. $\mathbf{A M L}=$ anterior mitral leaflet; AV = aortic valve; IVS = interventricular septum;.

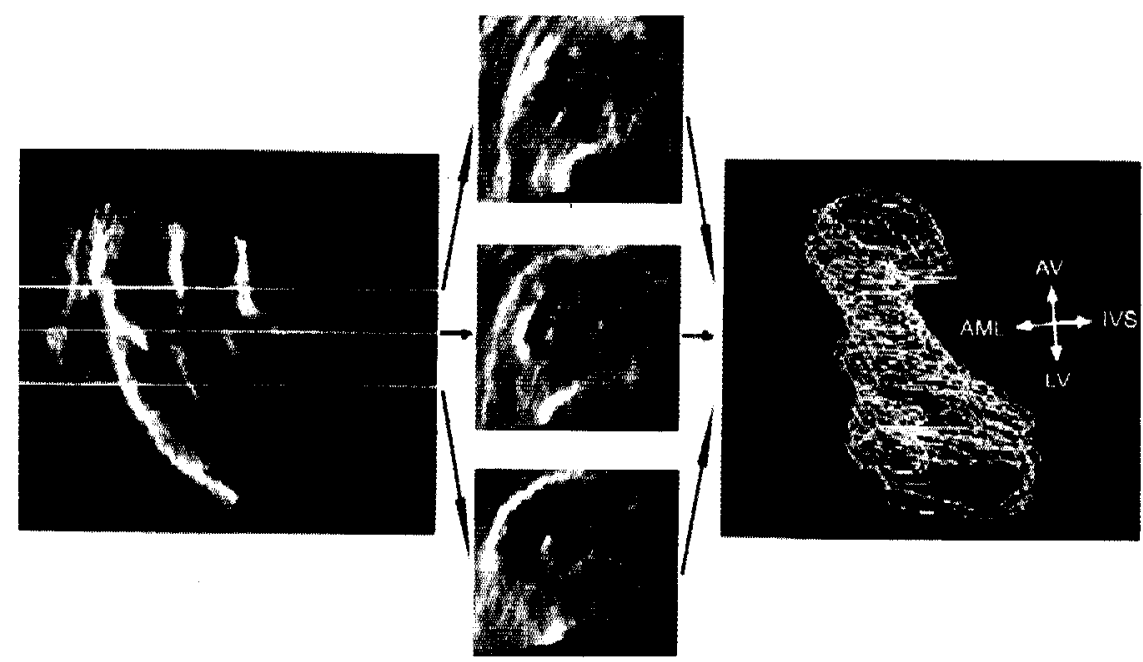

cially available system (Vingmed CFM 750 [Horten, Norway] or Toshiba Sonolayer SSH-140A [Tokyo, Japan]) equipped with a $3.5 \mathrm{MHz}$ transducer, while the patient was lying in the $45^{\circ}$ left recumbent position. After follow-up 2-dimensional echocardiographic study, the probe was positioned either at the left parasternal or apical window for acquisition of tomographic images of the LV outflow tract for 3dimensional reconstruction. Patient movement during the image acquisition can be prevented by thoroughly explaining the procedure before the study. The operator has to find the central axis of rotation so that the conical datasets encompass the LV outflow tract. During the acquisition, movements of the transducer holder must be avoided diligently. Mirror images of the first and the final cut-plane indicate a correct $180^{\circ}$ rotation. All subjects gave informed consent.

Three-dimensional echocardiography: IMAGE ACQUISITION: After the standard 2-dimensional examination, the video output of the echocardiographic system is interfaced to the acquisition system (Echo-scan, TomTec GmbH, Munich, Germany) and the transducer is fixed into a cylindrical holder. A step-motor mounted on this holder can rotate the probe around its longitudinal axis via a wheel-work interface. The step-motor is connected to the acquisition system which commands the rotation of the probe at $2^{\circ}$ intervals over a span of $180^{\circ}$. Respiratory and electrocardiographic gating are performed after the operator has selected the end-expiratory phase by thoracic impedance measurement and an adequate RR interval. Thus, only beats falling in the predetermined RR interval and at the end-expiratory phase are selected by the steering logic of the system and acquired. Cycles that do not meet the preset ranges are rejected. Ninety sequential cross-sections are acquired, each during a complete heart cycle, encompassing a 3-dimensional conical volume. After distance calibration, images are stored in the computer memory for subsequent analysis.

IMAGE PROCESSING: The raw data are resampled off-line according to their temporal and spatial lo- cation. The coordinates of each point are converted from a polar to a rectangular system, and the space between contiguous points is electronically filled with a trilinear cylindric interpolation. Several al gorithms are used to reduce noise and artifacts which can be created by patient and probe movements.

IMAGE DISPLAY ANALYSIS; Images were analyzed as follows:

1. After thorough examination of the 3-dimensional datasets with analysis of cardiac cross-sections in any desired plane ("anyplane echocardiography"), a cut-plane is selected where the LV outflow tract is visualized along its longitudinal axis.

2 . The onset of ventricular systole is selected, as the first frame during the cardiac cycle in which the mitral valve appears closed.

3. Electronic parallel slicing ("paraplane echocardiography") of the LV outflow tract perpendicular to the vertical axis is performed at $1 \mathrm{~mm}$ intervals, from the hinge point of the anterior mitral leaflet to the point of coaptation of the mitral leaflets, and the corresponding 2-dimensional images (both the stop frame at onset of systole and the dynamic sequence) are displayed.

4. On the stop frame image at the onset of systole, the endocardial contour of the cross-section is manually traced for automatic area measurement, and maximal and minimal diameters are also measured.

5. Finally, reconstruction of the LV outflow tract at the onset of ventricular systole is displayed in wire frame mode and the representative image can be rotated on the screen for versatile 3-dimensional evaluation (Figure 1).

From analysis of the 3-dimensional datasets, the following measurements of the LV outflow tract at the onset of systole were considered: (1) minimal cross-sectional area; (2) ratio between maximal and minimal cross-sectional area ( $\mathrm{max} / \mathrm{min}$ cross-sectional area), as an index of the "eccentricity" of the LV outflow tract (the higher the value, the greater the variations of the cross-sectional area throughout the length of the LV outflow tract; (3) ratio between maximal (lateromedial) and minimal (anteroposter- 


\begin{tabular}{|c|c|c|c|c|c|c|c|c|}
\hline Patient Number & Age $(y r) \&$ Sex & Type LVH & Therapy & Gradient & SAM & $\operatorname{CSA}\left(\mathrm{cm}^{2}\right)$ & Max/Min CSA & Max/Min Diameter \\
\hline 1 & $19 M$ & III & V & 58 & $3+$ & 2.9 & 2.0 & 1.9 \\
\hline 2 & $21 M$ & III & - & $<10$ & $2+$ & 2.4 & 2.5 & 1.7 \\
\hline 3 & $22 M$ & 1 & - & $<10$ & $1+$ & 3.0 & 1.8 & 1.4 \\
\hline 4 & $24 M$ & IV & - & $<10$ & $2+$ & 2.9 & 2.4 & 1.6 \\
\hline 5 & $25 \mathrm{M}$ & III & $\mathrm{V}$ & $<10$ & $2+$ & 2.7 & 2.4 & 1.6 \\
\hline 6 & $31 F$ & III & $S, V$ & 50 & $3+$ & 1.3 & 4.2 & 2.0 \\
\hline 7 & $38 \mathrm{~F}$ & II & V & $<10$ & 0 & 2.4 & 2.7 & 1.7 \\
\hline 8 & $39 \mathrm{~F}$ & III & V & $<10$ & 0 & 3.2 & 2.1 & 1.6 \\
\hline 9 & $41 \mathrm{M}$ & III & $\mathrm{V}$ & 20 & $1+$ & 1.9 & 1.7 & 1.8 \\
\hline 10 & $46 F$ & II & $A, V$ & $<10$ & $2+$ & 0.7 & 4.1 & 1.4 \\
\hline 11 & $53 M$ & III & - & $<10$ & $1+$ & 1.5 & 3.8 & 1.9 \\
\hline 12 & $63 M$ & $\|$ & $M$ & 100 & $3+$ & 1.5 & 3.0 & 2.6 \\
\hline 13 & $65 \mathrm{M}$ & III & V & 35 & $3+$ & 2.0 & 3.0 & 1.6 \\
\hline Mean \pm SD & $37 \pm 15$ & & & & & $2.2 \pm 0.7$ & $2.7 \pm 0.8$ & $1.75 \pm 0.3$ \\
\hline 14 & $31 M^{*}$ & III & - & $<10$ & 0 & 2.1 & 2.4 & 1.3 \\
\hline 15 & $34 M^{*}$ & III & S & $<10$ & $1+$ & 0.9 & 3.9 & 1.2 \\
\hline 16 & $44 M^{*}$ & lII & $S, V$ & $<10$ & 0 & 4.7 & 1.5 & 1.3 \\
\hline 17 & $59 \mathrm{M}^{*}$ & III & V & $<10$ & 0 & 3.0 & 1.6 & 1.3 \\
\hline Mean \pm SD & $42 \pm 12$ & & & & & $2.7 \pm 1.6$ & $2.3 \pm 1.1$ & $1.27 \pm 0.05$ \\
\hline Mean \pm SD (overall) & $39 \pm 15$ & & & & & $2.3 \pm 1.0$ & $2.6 \pm 0.9$ & $1.6 \pm 0.3$ \\
\hline
\end{tabular}

ior) diameter ( $\max / \mathrm{min}$ diameter) at the level of the cross-section with the minimal area, as an index of the "asymmetry" of the LV outflow tract (a ratio of 1 indicates a circular shape, with the highest values corresponding to the more elliptical shape of the cross sections).

Inter- and intraobserver reproducibility for the measurements of LV outflow tract with 3-dimensional echocardiography was assessed in all 27 patients. To assess interobserver variability, two observers (A.S. and Y.N.) independently measured the outflow tract area from the 3-dimensional datasets without prior knowledge of clinical data and without preselection of cut-planes. In addition, LV outflow tract cross-sectional area measurements were performed by 1 observer (A.S.) on 2 occasions (3 months apart, without preselection of cut-planes from the 3-dimensional datasets) to assess intraobserver variability.

Statistical analysis: Values are given as mean \pm SD. Student's unpaired $t$ test was used to compare the differences between $\mathrm{HC}$ and control subjects. Values of $p<0.05$ were considered to be significant. Reproducibility of the LV outflow tract measurements was expressed in terms of mean differences and $95 \%$ confidence intervals. ${ }^{12}$

\section{RESULTS}

Three-dimensional acquisition could be performed successfully in all patients. Echocardiographic acquisition of the image of the LV outflow tract for 3-dimensional reconstruction was performed either from the parasternal $(n=20)$ or apical $(n=7)$ windows, according to the image quality. The examination including the calibration procedure, selection of the optimal axis of rotation, a num- ber of test runs, and the actual image acquisition required approximately 10 minutes in addition to the time required for the standard 2-dimensional echocardiogram. Three-dimensional reconstruction of the images was possible and of good quality in all patients. The time required for post-processing the raw data, and reconstruction and analysis of the images was approximately 20 minutes. Demographics, echocardiographic characteristics, and measurements of the LV outflow tract in each patient with $\mathrm{HC}$ are reported in Table I.

Left ventricular outflow tract in patients with hypertrophic cardiomyopathy versus normal subjects: CROSS-SECTIONAL AREA: The values of minimal cross-sectional area of the individual subjects are plotted in Figure 2. The minimal LV outflow tract crosssectional area calculated with 3-dimensional echocardiography was significantly smaller in patients with $\mathrm{HC}$ than in the control group (2.3 \pm 1.0 vs $\left.5.0 \pm 0.9 \mathrm{~cm}^{2}, p<0.0001\right)$. Thirteen of the 17 patients with $\mathrm{HC}$ had a value smaller than controls, and 2 of the $4 \mathrm{HC}$ patients (nos. 6 and 17) with higher values were evaluated after myectomy. After correction for body surface area the values were $1.3 \pm 0.5$ and $2.7 \pm 0.6 \mathrm{~cm}^{2}$, respectively $(\mathrm{p}<0.0001)$.

SHAPE: From the 3-dimensional datasets, the reconstructed LV outflow tract could be displayed as observed from different viewpoints. This simplifies visualization of the geometry and shape as well as the localization of the narrowing of the LV outflow tract in patients with HC (Figure 3). A similar display in a normal patient is shown in Figure 4. Some examples of 3-dimensional reconstruction of the LV outflow tract in normal subjects and in patients with $\mathrm{HC}$ are shown in Figure 5. 


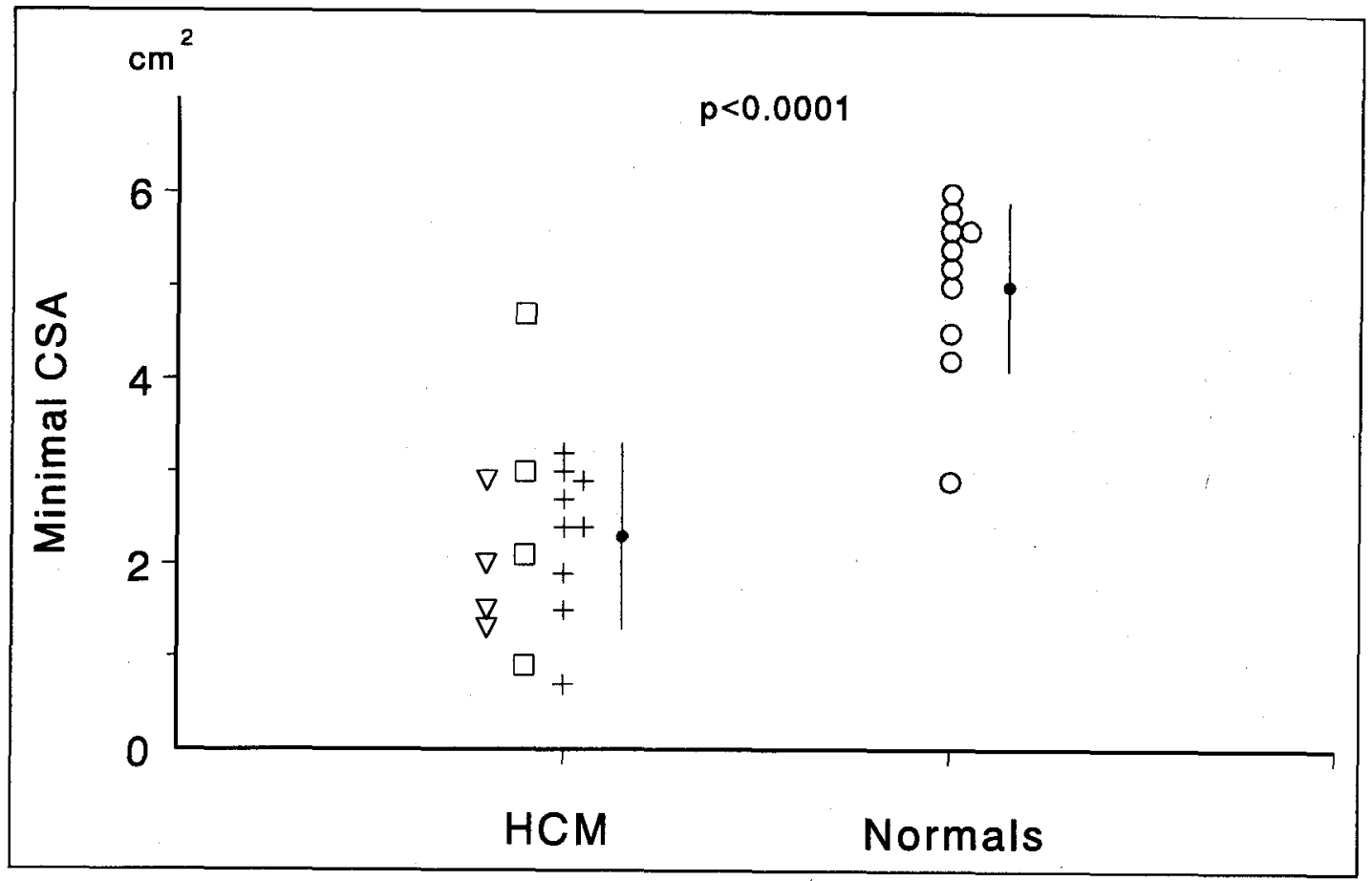

FIGURE 2. Minimal cross-sectional area (CSA) of the left ventricular outflow tract in patients with hypertrophic cardiomyopathy (HCM) and in normal subjects $(O) .+=$ patients without obstruction; $\square=$ patients after myectomy; $\nabla=$ patients with obstruction.

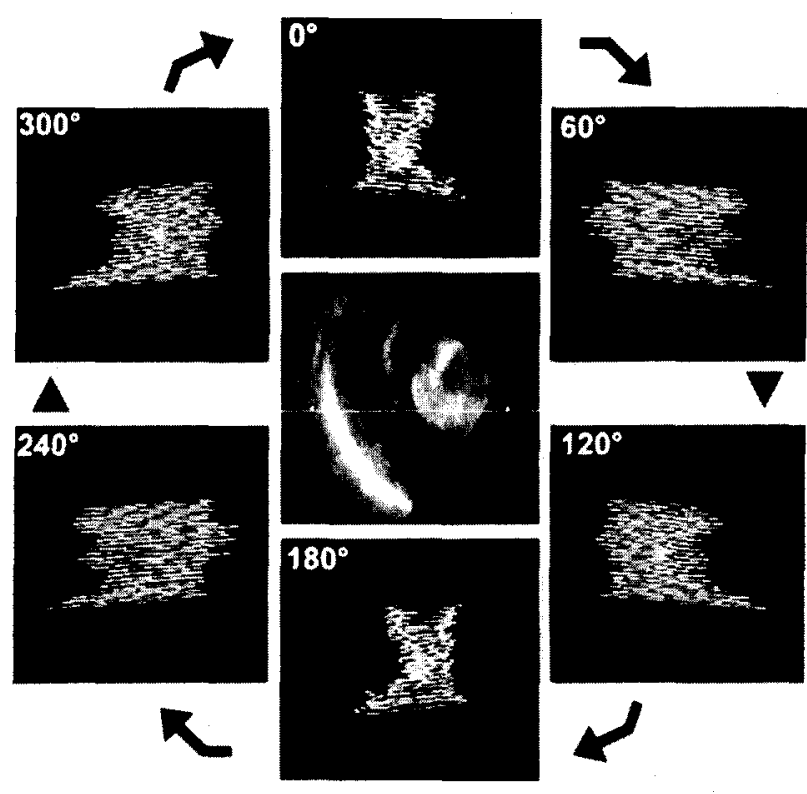

FIGURE 3. In this patients with hypertrophic cardiomyopathy, the wire frame display of the reconstructed left ventricular (IV) outflow tract is represented as observed from different viewpoints. The view at $0^{\circ}$ corresponds to the cut-plane represented in the central panel. The images obtained after incremental $60^{\circ}$ clockwise rotation are displayed in the corresponding panels. There is an eccentric and asymmetric shape of the LV outflow tract. From these images it is also apparent that the narrowing is localized at the middle-caudad part of the LV oufflow tract and is mainly related to a reduction in the anteroposterior diameter.

MAX/MIN CROSS-SECTIONAL AREA (ECCENTRICITY INDEX): The max/min cross-sectional area of the $\mathrm{LV}$ outflow tract derived from the 3-dimensional datasets are displayed in Figure 6. From this figure, it is

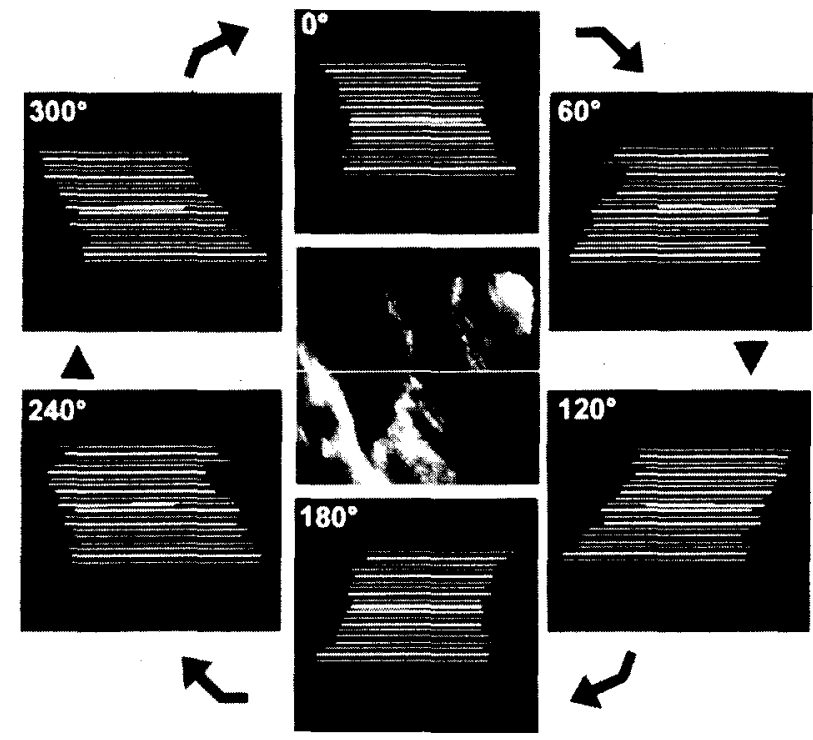

FIGURE 4. Three-dimensional reconstruction of the left ventricular (LV) outflow tract in a normal subject, with the same display as in Figure 3. Note the uniformity (indicated by the minimal variations of the diameters throughout the length of the LV oufflow tract) as well as the symmetry (indicated by the similar diameters of individual cross-sections from different viewpoints) of the LV outflow tract.

apparent that patients with $\mathrm{HC}$ had higher ratios $(2.6$ \pm 0.9 ), with a broad range of values (from 1.5 to 4.2 ) indicative of many irregular different shapes of the LV outflow tracts. In contrast, normal subjects had smaller ratios $(1.4 \pm 0.2)$, with a narrow range of values (from 1.1 to 1.6). In particular, each of the controls had a ratio of $\leq 1.6$, whereas 15 of 17 patients with $\mathrm{HC}$ had a ratio of $>1.6$. The 2 patients 

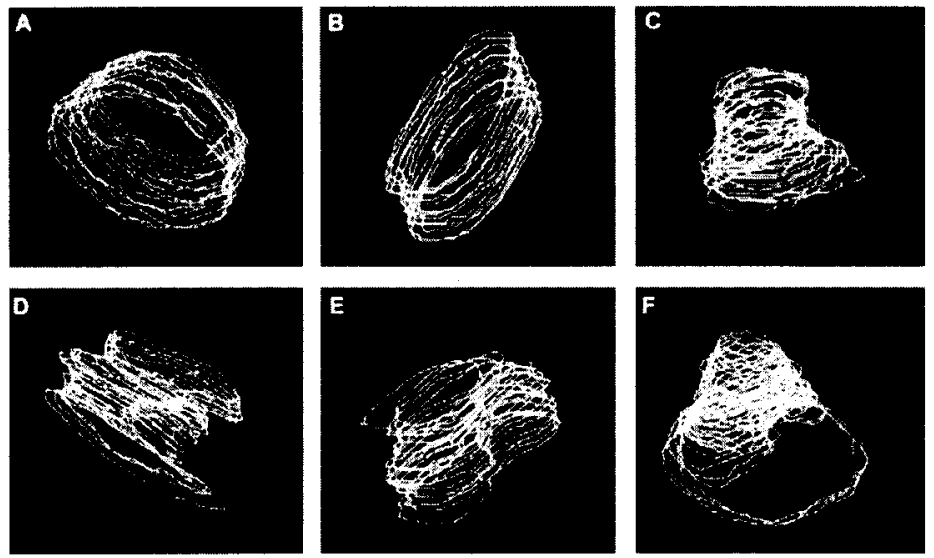

FIGURE 5. Three-dimensional reconstruction of the left ventricular outflow tract with wire frame display in normal subjects $(A$ to $C)$ and in patients with hypertrophic cardiomyopathy ( $D$ to $F$ ). The different irregular configuration of the left ventricular oufflow tract in patients with hypertrophic cardiomyopathy can be evaluated.

with $\mathrm{max} / \mathrm{min}$ cross-sectional area of $\leq 1.6$ (patients 16 and 17) were evaluated after myectomy. Thus, an outflow tract area ratio of 1.6 appeared to separate patients with from subjects without HC.

MAX/MIN DIAMETER (ASYMMETRY INDEX): The individual values of $\max / \mathrm{min}$ diameter of the LV outflow tract cross-section are displayed in Figure 7. This ratio was significantly higher in patients with $\mathrm{HC}$ than in normal subjects $(1.6 \pm 0.3$ vs $1.2 \pm 0.1, \mathrm{p}=$ 0.001 ). Of interest, patients evaluated after myectomy had the lowest values; conversely, the highest values were found in patients with $\mathrm{HC}$ and LV outflow tract obstruction. An index of 1.36 appeared to separate normal subjects from patients with $\mathrm{HC}$ who did not undergo operation.

Reproducibility analysis of three-dimensional echocardiography: INTEROBSERVER VARIABILITY: The difference between the 2 observers for measurements of the LV outflow tract was compared with the average of the 2 measurements for each patient. The mean difference between the measurements of the 2 observers was $0.04 \mathrm{~cm}^{2}$ ( $95 \%$ confidence interval -0.08 to 0.12 $\mathrm{cm}^{2}$ ) for cross-sectional area.

INTRAOBSERVER VARIABILITY: The difference between the 2 measurements made by the same observer was compared with the average of the 2 measurements for each patient. The mean difference between the 2 measurements was $0.13 \mathrm{~cm}^{2}$ (95\% confidence interval -0.01 to $0.25 \mathrm{~cm}^{2}$ ) for crosssectional area.

\section{DISCUSSION}

Hypertrophic cardiomyopathy is a disease with a great individual variability and "no two hearts are alike." ${ }^{13}$ The results of the present study indicate that 3-dimensional echocardiography allows visualization of the varied complex geometry of the LV outflow tract in patients with HC. With quantitative analysis of the 3-dimensional datasets, we demonstrated that in patients with $\mathrm{HC}$ the minimal crosssectional area is smaller than that in normal subjects. In addition, most patients with $\mathrm{HC}$ have an irregular shape of the LV outflow tract as demonstrated by an eccentricity index of $\geq 1.5$. In normal subjects, this

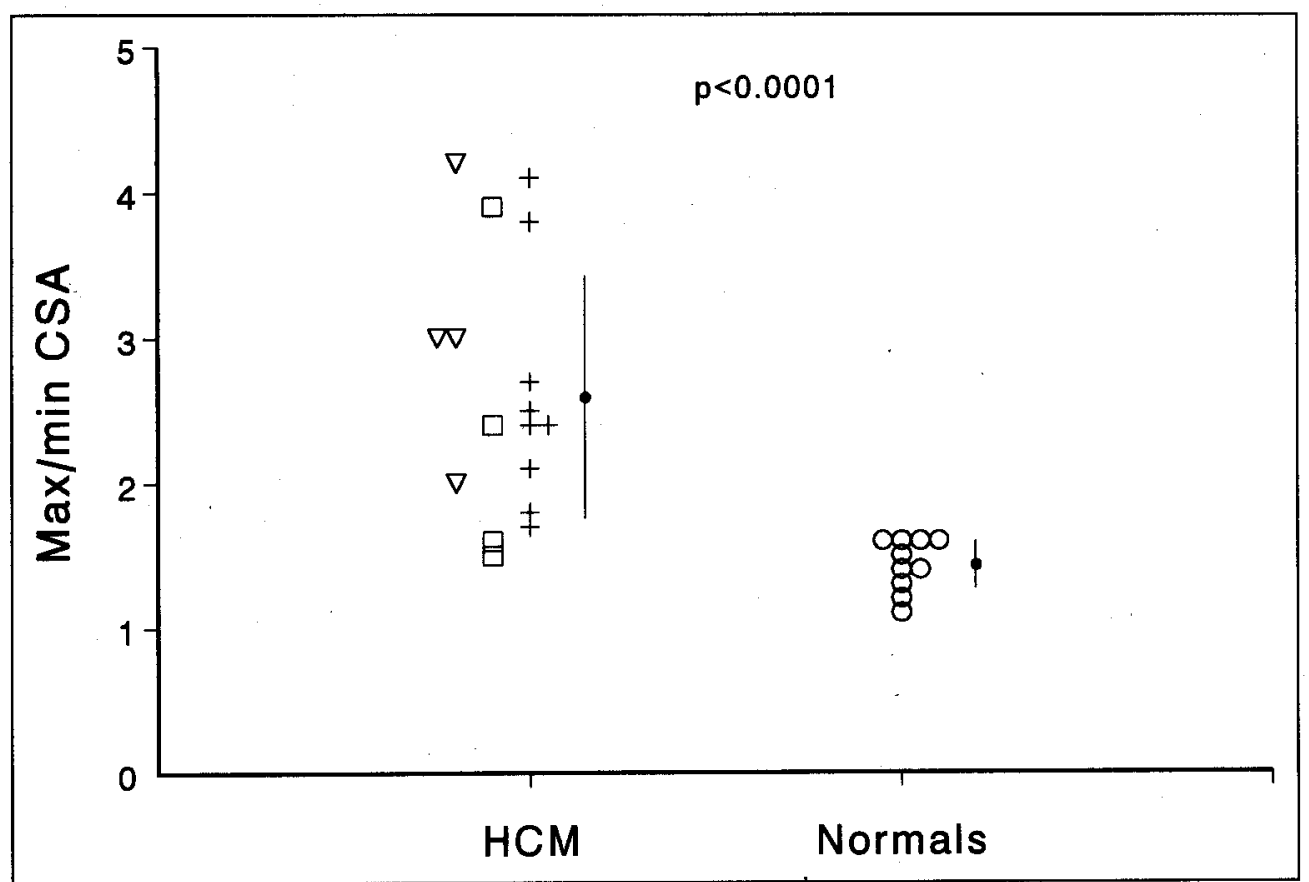

FIGURE 6. Ratio between maximal and minimal cross-sectional areo (max/min CSA) of the left ventricular outflow hract in patients with hypertrophic cardiomyopathy (HCM) and in normal subjects. A high ratio indicates an eccentric shape of the left ventricular outHow tract. Symbols as in Figure 2. 


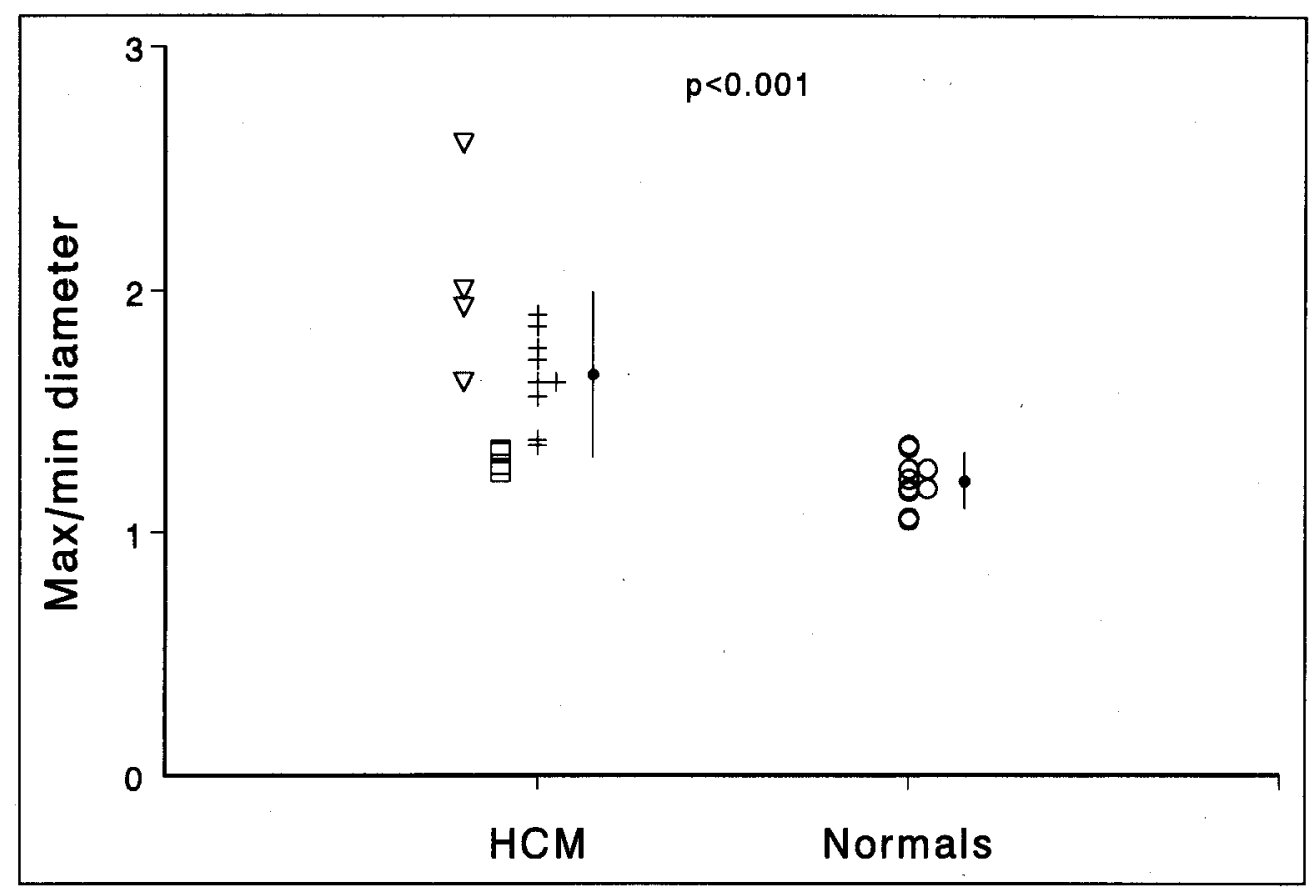

FIGURE 7. Ratio between maximal and minimal diameter (max/min diameter) measured at the cross-section of the left ventricular oufflow tract with the minimal area in patients with hypertrophic cardiomyopathy (HCM) and in normal subjects. A high ratio indicates an asymmetric shope of the left ventricular outflow tract. Symbols as in Figure 2.

index is always $\leq 1.6$, indicating a uniform shape of the LV outflow tract, without significant variation of the cross-sectional area throughout its length. We have also demonstrated that the cross-sectional shape of the LV outflow tract is more elliptical in patients with $\mathrm{HC}$ than in normal subjects, as indicated by a higher ratio of $\mathrm{max} / \mathrm{min}$ diameter measured at the plane of the minimal cross-sectional area. This finding is in agreement with the concept that in $\mathrm{HC}$ the hypertrophic ventricular septum narrows the LV outflow tract mainly along its anteroposterior diameter. Of interest, this asymmetry index was highest in patients with $\mathrm{HC}$ and obstruction of the LV outflow tract at rest. In contrast, in $\mathrm{HC}$ neither the minimal cross-sectional area nor the eccentricity index of the LV outflow tract separated this subgroup. This finding indicates that for similar cross-sectional area the asymmetry of the LV outflow tract plays an important role in determining the presence of significant obstruction at rest.

Patients who had undergone myectomy had a minimal cross-sectional area similar to other patients with $\mathrm{HC}$, including those with obstruction (Figure 2 ). However, from Figure 7 it is clear that after myectomy the asymmetry index was lowest, indicating that the surgical remodeling of the LV outflow tract was adequate for the relief of the obstruction despite the finding that the cross-sectional area remained small compared with that in normal controls, and remained in the same range as that of the other $\mathrm{HC}$ patients without previous myectomy. Thus, in patients with $\mathrm{HC}$, precordial 3-dimensional echocardiography has the potential to play a major role in tailoring the standard surgical resection of the interventricular septum to the individual patient's anat- omy, which is crucial for safe and efficacious performance of myectomy, and also for evaluation of the results of surgery.

Evaluation of left ventricular outflow tract using threedimensional echocardiography: Previous experience with 3-dimensional echocardiography for the evaluation of the LV outflow tract was based on morphologic analysis with volume-rendered display. ${ }^{14}$ In contrast, for quantitative analysis of the LV outflow tract performed in the present study, we selected a multitude of cut-planes ("anyplane echocardiography"') and performed a parallel scanning of the LV outflow tract at $1 \mathrm{~mm}$ intervals ("paraplane echocardiography"). This rate of sampling of the dataset is similar to the analysis done with magnetic resonance imaging or computed tomography, and allows detailed spatial information, ${ }^{15}$ While some display modalities, such as volume rendering, are indicated more for representation of anatomic details, ${ }^{16-18}$ the wire frame display format appears particularly suited to 3-dimensional reconstruction of the cardiac cavities, where areas, volumes, size, and shape can be adequately evaluated.

Limitations of three-dimensional echocardiography: In this study, patients were selected on the basis of high-quality images at 2-dimensional echocardiography, which yielded a success rate of 3-dimensional reconstruction of $100 \%$. The same results cannot be expected from an unselected population where poor quality precordial images may prevent adequate quality of the reconstruction.

Echocardiographic images were acquired by an experienced technician and reconstruction was performed by a cardiologist after a learning period of $>50$ studies. This previous experience of 3-dimen- 
sional echocardiography prevented artifacts in the 3dimensional datasets, limited the time required both for acquisition and reconstruction, and resulted in a minimal variability for the measurements.

Acknowledgment: The expert technical assistance of Renè Frowijn, BSc, in the preparation of the illustrations is greatly acknowledged.

1. Henry WL, Clark CE, Roberts WC, Morrow AG, Epstein SE. Difference in distribution of myocardial abnormalities in patients with obstructive and nonobstructive asymmetric septal hypertrophy (ASH). Echocardiographic and gross anatomic findings. Circulation 1974;50:447-455

2. Maron BJ, Bonow RO, Cannon RO III, Leon MB, Epstein SE. Hypertrophic cardiomyopathy. Interrelations of clinical manifestations, pathophysiology, and therapy. (First of two parts). N Engl J Med 1987;316:780-789.

3. Panza JA, Maris TJ, Maron BJ. Development and determinants of dynamic obstruction to left ventricular outflow in young patients with hypertrophic cardiomyopathy. Circulation 1992;85:1398-1405.

4. Klues HG, Roberts WC, Maron BJ. Morphological determinants of echocardiographic patterns of mitral valve systolic anterior motion in obstructive hypertrophic cardiomyopathy. Circulation 1993;87:1570-1579.

5. Spirito P, Maron BJ. Significance of left ventricular outflow tract crosssectional area in hypertrophic cardiomyopathy: a two-dimensional echocardiographic assessment. Circulation 1983;67:1100-1108.

6. McIntosh CL, Maron BJ. Current operative treatment of obstructive hypertrophic cardiomyopathy. Circulation 1988;78:487-495.
7. Salustri A, Roelandt JRTC. Three-dimensional reconstruction of the heart with rotational acquisition: methods and clinical applications. Br Heart $J$ 1995;73 (suppl 2):10-15.

8. Roelandt JRTC, Salustri A, Bekkering L, Bruining N, Vletter WB. Precordial three-dimensional echocardiography with a rotational imaging probe: methods and initial experience. Echocardiography 1995;12:243-252.

9. Salustri A, Spitaels S, McGhie J, Vletter W, Roelandt JRTC. Transthoracic three-dimensional echocardiography in adult patients with congenital heart disease. J Am Coll Cardiol 1995:26:759-767.

10. Maron BJ, Epstein SE. HCM: a discussion of nomenclature. Am J Cardiol 1979;43:1242-1244.

11. Maron BJ, Gottdiener JS, Epstein SE. Patterns and significance of distribution of left ventricular hypertrophy in hypertrophic cardiomyopathy. A wideangle, two-dimensional echocardiographic study of 125 patients. Am J Cardiol $1981 ; 48: 418-428$.

12. Bland JM, Altman DG. Statistical method for assessing agreement between two methods of clinical measurement. Lancet 1987;1:307-310.

13. Roberts CS, Roberts WC. Morphologic feature. Prog Cardiol 1989;2(pt 2):3-32. 14. Fyfe DA, Ludomirsky A, Sandhu S, Dhar PK, Silberbach M, Sahn DJ. Left ventricular outflow tract obstruction defined by active three-dimensional echocardiography using rotational transthoracic acquisition. Echocardiography 1994;11:607-615.

15. Salustri A, Roelandt JRTC. Left atrial myxoma visualized by transesophageal Echo-CT. Circulation 1995;91:2290.

16. Roelandt JRTC, ten Cate FJ, Vletter WB, Taams MA. Ultrasonic dynamic three-dimensional visualization of the heart with a multiplane transesophageal imaging transducer. $J A m$ Soc Echocardiogr 1994;7:217-229.

17. Pandian NG, Roelandt J, Nanda NC, Sugeng L, Cao QL, Azevedo J, Schwartz SL, Vannan MA, Ludmirski A, Mary G, Vogel M. Dynamic threedimensional echocardiography: methods and clinical potential. Echocardiography 1994;11:237-259.

18. Schwartz SL, Cao Q, Azevedo J, Pandian NG. Simulation of intraoperative visualization of cardiac structures and study of dynamic surgical anatomy with real-time three-dimensional echocardiography. Am J Cardiol 1994;73:501-507. 